\title{
Stochastic Lotka-Volterra equations: a model of lagged diffusion of technology in an interconnected world
}

\author{
Anindya S. Chakrabarti * \\ Economics area, Indian Institute of Management \\ Ahmedabad 380015, India
}

September 2, 2015

\begin{abstract}
We present a model of technological evolution due to interaction between multiple countries and the resultant effects on the corresponding macro variables. The world consists of a set of economies where some countries are leaders and some are followers in the technology ladder. All of them potentially gain from technological breakthroughs. Applying Lotka-Volterra (LV) equations to model evolution of the technology frontier, we show that the way technology diffuses creates repercussions in the partner economies. This process captures the spill-over effects on major macro variables seen in the current highly globalized world due to trickle-down effects of technology.
\end{abstract}

\section{Introduction}

Economies are perfect examples of complex systems which give rise to patterns out of interaction between smaller economic entities. Traditionally econophysics literature has mostly been concerned about studies of a specific set of empirical regularities. A part of the literature focuses on the empirical validation of those regularities and the other part concerns the theoretical origin of the same. The inverse cubic law of stock prices, Zipf's law of income and wealth distributions, scaling behavior of volatility of economic entities like firms or countries with respect to their sizes are examples of the first stream of the literature (see Ref. [1] for statistical description of the stock markets and see Ref. [2] for a general introduction to the econophysics literature). Generally it has been difficult to establish empirical regularities in an useful sense because the moment one finds a generally recognizable empirical regularity, people start working on the basis of it undoing the premise in the meantime. This is especially true of the stock market although as has been mentioned before, some features like the inverse cubic law are extremely robust. If one considers macroeconomic scenarios, regularities are scarce. Apart from the size distributions of certain variables (like firm-size),

*Email address: anindyac@iimahd.ernet.in 
there do not exist many other robust and stable features. It is also difficult to extract the effects of such distributions on the economy at the macro level ${ }^{1}$.

In this paper, we ask how one can model the complex interdependence of macro variables across the countries. The countries in the modern globalized world have nonlinear dependence on each other. This becomes apparent from the growth predictions of countries, which are extremely susceptible to the economic situation in the rest of the world. It is also clear that the regime of linear cause-effect relationship is often not a good approximation, be it for a set interdependent of banks or countries embedded in a network of trade and capital or technology flow. After the last economic crisis, the attention has been shifted away from interaction between well behaved homogeneous economic entities. Different sources of heterogeneity manifested via distribution of connectivity or size have been seen to play important roles in shaping up macro scenario. Spill-over effects and the question of correlation has been framed in multiple formats, especially in the network literature (see for example, Ref. [4-11]).

In order to model such interdependence of macro variables, we rely on a technique borrowed from the literature on theoretical ecology (Ref. [12-14]). The famous Lotka-Volterra system (LV henceforth) has been used for long to model predator-prey relationship among different species. This type of model have been used extensively for modeling stock market behavior. Ref. [15] (as well as Ref. [16,17]) applied it to model interaction of heterogeneous agents. Ref. [18] studies the concept of dynamical stability in a large system of heterogeneous agents placed on random networks (see also Ref. [19]). In the present context, we model technological evolution assuming that some countries are predators and some countries are preys. The prey countries spend resources to develop newer technology whereas the predators use the same without spending resources diminishing the advantage of usage which hurts relative productivity of the prey countries. An interesting feature of this kind of models is that the prey population always leads the predator population (Ref. [20]). This is automatically translated in the economic context as when a big technology boom occurs in the leading countries, the followers borrow them subsequently enabling them to gain advantage. But they run with a lag always because of the dependency on the leading countries.

The theoretical argument has been forwarded by Ref. [21] where it is argued that the equilibrium distribution of technology might be highly asymmetric, resulting in different levels of technological advancement across countries. The 'cut-throat' capitalist economies would spend resources to become technology leaders and the rest, termed as 'cuddly' capitalists, would follow their lead. They cannot be on the technology frontier but this loss is offset by advantage of spending lesser resources on research and development, which leads to lesser within-economy inequality. The major insight coming from such a formulation is that, to maximize aggregate welfare (or utility) at the global level it is best to have both types of countries. If leaders themselves become followers, that will reduce welfare. Similarly for followers to become leaders, they must sacrifice welfare resulted in by increasing inequality. Thus an important property of the economic system is that the evolution of competing countries ensured global maximization of utility without any global coordination between them.

\footnotetext{
${ }^{1}$ However, in recent times there has been a major advancement in understanding the effects of fat tails on the aggregate volatility of the economy. See for example Ref. [3].
} 
From the aforementioned work, the idea of leaders and followers interacting on the technology frontier is borrowed and we show that coupled with the business cycle literature, it generates time-series with certain properties that closely resemble the real data. Interestingly, Ref. [22] studied diffusion of technology at the micro-level and showed that innovation and imitation are important in the mechanics of knowledge diffusion. Here we do not provide any microeconomic argument about what causes the diffusion. Instead we opt for a description of the process at the macro level which is in line with the micro evidence. The individual time-series properties of both the leader and the follower show substantial autocorrelation with alternating signs even with relatively large lags. This feature has been studied by multiple papers in the business cycle literature. Ref. [23] studied a two-sector model with sector-specific increasing returns to scale which is able to mimic such a behavior. Ref. [24] shows that a labor adjustment cost in an otherwise standard real business cycle model generates positive autocorrelation after several lags. In general, a problem has been that this sort of models require implausible parameter values to match data well. Thus there is little consensus about the correct specification of such models. The model that we study in the following has no internal propagation mechanism. Rather it inherits all the properties of the exogenous shocks which means output series essentially mimics the shock process. The optimization mechanism does not impose any extra properties on the output series. The usefulness of the LV type specification is that it readily captures many of the time-series properties of real data and hence, the response of the macro variables show similar behavior, in turn.

In recent times, questions related to growth of countries (or other economic entities like, firms for example) received prominence in the econophysics literature. Ref. [25] argues that wealth of a country is directly related to the competitiveness of the same. Ref. [26] argues that level of wealth is also related to asymmetric effects of price changes on volatility (see also Ref. [27-29]). Ref. [30] studied decomposition of macroeconomic volatility in G-7 countries and Ref. [31] studied other time-series properties including persistence of a similar

set of variables. To complement such analysis, we need a general framework to address the interdependence in a business cycle model.

\section{The model}

Below I describe a two-country model by combining the real business cycle literature and the species competition literature to elucidate how we can generate a complex structure of cross-correlation and autocorrelation in the macroeconomic variables at the aggregate level. The small scale real business cycle (popularly known as the rbc models) type of models are extremely useful to neatly explain the comovements between the major variables. The LV equations are used to model evolution of the technology frontier which is formed by continuous innovation, copy and disposition of technology by the competing countries. For simplicity, one can imagine measuring technology by the number of blue-prints. A bigger set of blue-prints are useful as it raises productivity of labor. The interaction arises because some countries are predators and some countries are prey in this scenario. A predator might feed on higher number of blue-prints generated by the prey and by dissolving the use of the same blue-prints, they become obsolete. Thus the predator follows the prey on the technology 
frontier.

Let us denote these two economies by $L$ (leader) and $F$ (follower). Time is discrete and goes till infinity. The underlying structure of $L$ and $F$ are identical. Both admits an unit mass of households that provide labor and lends capital to produce output which they either consume or save and invest. There are many expositions of such models available. See for example, Ref. [32] for a continuous time formulation or Ref. [33] for a discrete time formulation. We follow the latter as a discrete-time formulation is more suitable for finding the time-series behavior. Following literature, we assume that there is only one good produced in each economy. In general, in these models there are a number of agents who provide labor to produce output, earn wage in return and consume goods. Usually they are considered to be maximizing their objective function (utility) defined over consumption. There are firms that buy labor input (and pay wage to the workers) and combine it with physical capital to produce output. They are also maximizing their own objective function (profit). Finally, capital is produced by the savings (income-consumption) of the workers. This closes the whole model. In the next section, we describe the idea mathematically.

\subsection{Households and firms}

The utility function in the $j$-th country $(j \in\{L, F\})$ is given by the standard form

$$
U^{j}=\sum_{t=0}^{\infty} \beta^{t}\left(\ln c_{t}^{j}+\alpha \ln \left(1-l_{t}^{j}\right)\right),
$$

where $c_{t}^{j}$ and $l_{t}^{j}$ are consumption and labor in period $t$ respectively. Maximum amount of labor per unit of time is normalized to 1 for simplicity. The parameter $\beta$ denotes the subjective discount rate over time. The other parameter $\alpha$ controls the relative weight assigned to leisure with respect to consumption. The production function has a standard functional form with a stochastic technology shock,

$$
y_{t}^{j}=z_{t}^{j}\left(k_{t}^{j}\right)^{\theta}\left(l_{t}^{j}\right)^{1-\theta},
$$

which basically says that output $y_{t}^{j}$ is produced by combining capital $k_{t}^{j}$, labor $l_{t}^{j}$ and a technology variable $z_{t}^{j}$. The productivity shocks are captured by the term $z_{t}^{j}$ which is stochastic. Both the utility and production functions have the simplest forms. One can work with more complicated forms, but with little value addition. Capital accumulation is done by investment in the usual way,

$$
k_{t+1}^{j}=(1-\delta) k_{t}^{j}+i_{t}^{j},
$$

where $\delta$ is the rate of depreciation and $i_{t}$ is the investment. Finally, we have a resource constraint that output is either consumed or invested,

$$
c_{t}^{j}+i_{t}^{j} \leq y_{t}^{j} .
$$

The evolution of $z_{t}^{j}$ in the production function (Eqn. 2) is of particular importance to us. In the standard description of this type of models, $z_{t}^{j}$ is usually assumed to represent a technological shock process independent of the rest of the economy. Here, we assume that the technology evolves jointly across countries. This is where the LV type interacting specie model comes in, as we describe below. 

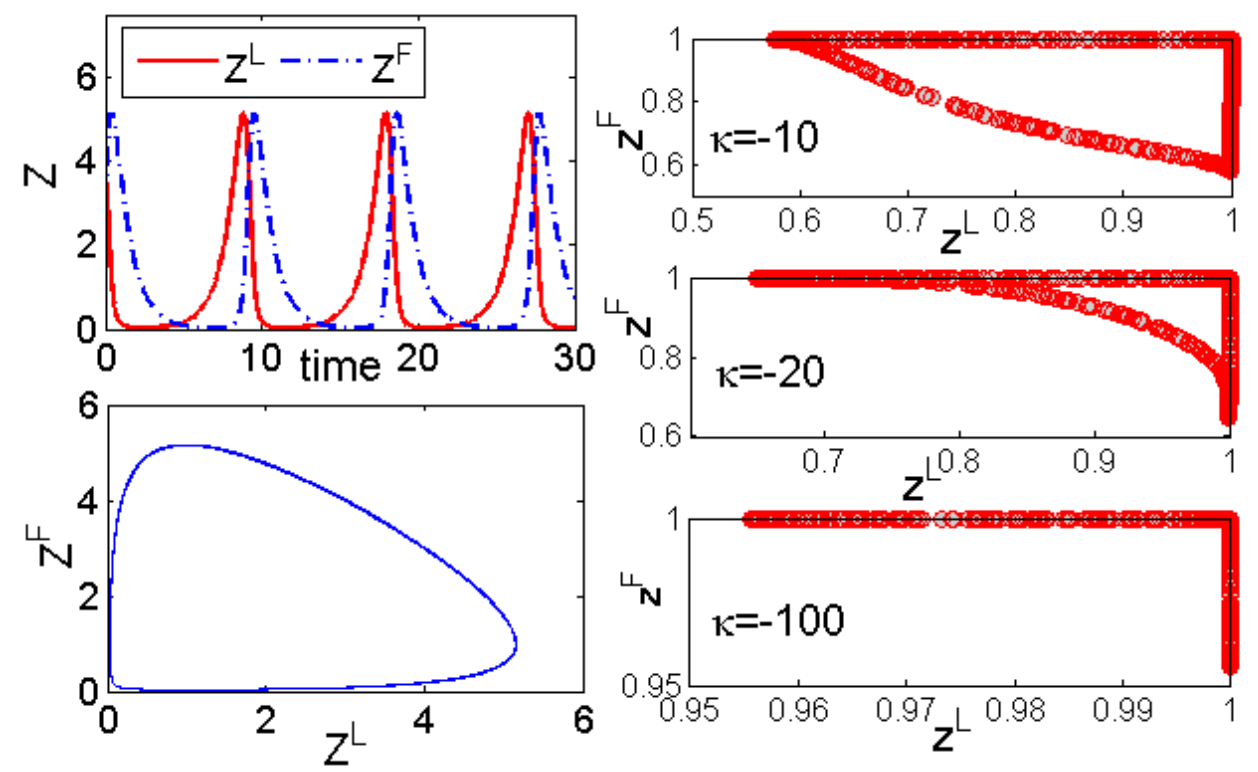

Figure 1: Evolution of a canonical LV model. The upper-left panel shows technology evolution in both countries. The lower-right panel shows the locus of the solution. On the right column three insets show the same trajectory under a functional transformation following Eqn. 7 with three different values of the tuning parameter, $\kappa=-10,-20$ and -100 .

\subsection{Evolution of the technology frontier}

The technology frontier is represented in continuous time by the generic model of speciescompetition viz.,

$$
Z^{\prime}=G(Z)
$$

where $Z$ is a vector $\left\{Z^{L}, Z^{F}\right\}$ and $G$ is the interaction matrix. The evolution of $Z^{L}$ (or $Z^{F}$ ) represents the evolution of the state of technology over time of the leading (following) economy. This is a continuous process. However, the households and firms see a snapshot of it in certain times and respond to that level of technology, which we denote by $\left\{z^{L}, z^{F}\right\}$.

We impose a specific structure on the interaction matrix $G$ assuming that the underlying technology evolves as follows:

$$
\begin{aligned}
& \frac{d Z^{L}}{d t}=\mu_{1} Z^{L}-\mu_{2} Z^{L} Z^{F} \\
& \frac{d Z^{F}}{d t}=-\mu_{3} Z^{L}+\mu_{4} Z^{L} Z^{F}
\end{aligned}
$$

where $\mu_{\{1, \ldots, 4\}}$ are parameters. This basic structure admits analytical solution but for our purpose, we will focus on a stochastic sequence (and an algebraic transformation thereof) of the time-path generated by the model. We assume that the agents sample it at random intervals (see App. 5.3.3 for details). The discrete sequence that they observe is denoted by $\left\{S_{\tau}\right\}=\left\{Z^{L}(\tau), Z^{F}(\tau)\right\}$ where $\tau=1,2,3, \ldots$ This process is discrete as we require by our 
discrete time formulation of the economy, but has high variance. To have a well-behaved process with tunable variance, we make one transformation to describe the shock process as

$$
z^{j}(t)=\frac{1}{1+e^{-\kappa S^{j}(t)}} \quad j \in\{L, F\}
$$

where $\kappa$ is a constant controlling the spread of the technology variables $\left\{z^{j}() t\right\}$ for $j \in\{L, F\}$. Such a transformation reduces volatility of the process making it a suitable candidate for studying the behavior close to the steady state of the model (which is dictated by Eqn. 8 below). Fig. 1 shows the evolution of a canonical LV model. The insets show that the high variance process can be mapped into a process with much lower variance by the transformation considered with an appropriate value of the tuning parameter $\kappa$. The leader is termed prey and the follower is termed predator in the literature of theoretical ecology. Here the metaphor works as the technological booms and busts in the leading economy is followed by the other economy with a lag. Thus essentially we model a 'trickle-down' version of technological evolution. We elaborate on the macroeconomic interpretation of this feature of the model in Sec. 3.1. For simulation purpose, we have assumed that $\mu_{j}=1$ for $j \leq 4$ (initial value pair is 4,3). Fig. 4 shows the cross-correlation and the autocorrelation structure of the technology path followed by the predator and prey countries.

\subsection{Solving the model}

We can easily find out the first order conditions by solving the dynamic programing problem of maximizing utility subject to the budget constraint (see Ref. [33] and the references therein). The whole system of equations that captures the evolution of the economy subject to technology shocks are described as follows,

$$
\begin{aligned}
\frac{1}{C_{t}^{j}} & =\beta E_{t}\left(\frac{1}{C_{t+1}^{j}}\left(r_{t+1}^{j}+1-\delta\right)\right), \\
\alpha C_{t}^{j} & =(1-\theta)\left(1-L_{t}^{j}\right) \frac{Y_{t}^{j}}{L_{t}^{j}}, \\
C_{t}^{j}+K_{t+1}^{j} & =Y_{t}^{j}+(1-\delta) K_{t}^{j}, \\
Y_{t}^{j} & =z_{t}^{j}\left(K_{t}^{j}\right)^{\theta}\left(L_{t}^{j}\right)^{1-\theta}, \\
r_{t}^{j} & =\theta\left(\frac{Y_{t}^{j}}{K_{t}^{j}}\right) .
\end{aligned}
$$

Note that we have replaced the agent specific variables by aggregate variables as within country heterogeneity is irrelevant in the present context. The equations are respectively (1) Euler equations (showing trade-off between consumption today and tomorrow), consumptionleisure trade-off (should I consume more or enjoy leisure today?), aggregate resource constraint (all production is either consumed or invested), production function (outputs are produced by combining labor and capital) and the interest rate. Wage rate can also be pinned down from the same. See App. 5.3.3 for a detailed algorithm to solve the model in conjunction with the LV mechanism and find the time-series generated by it. 

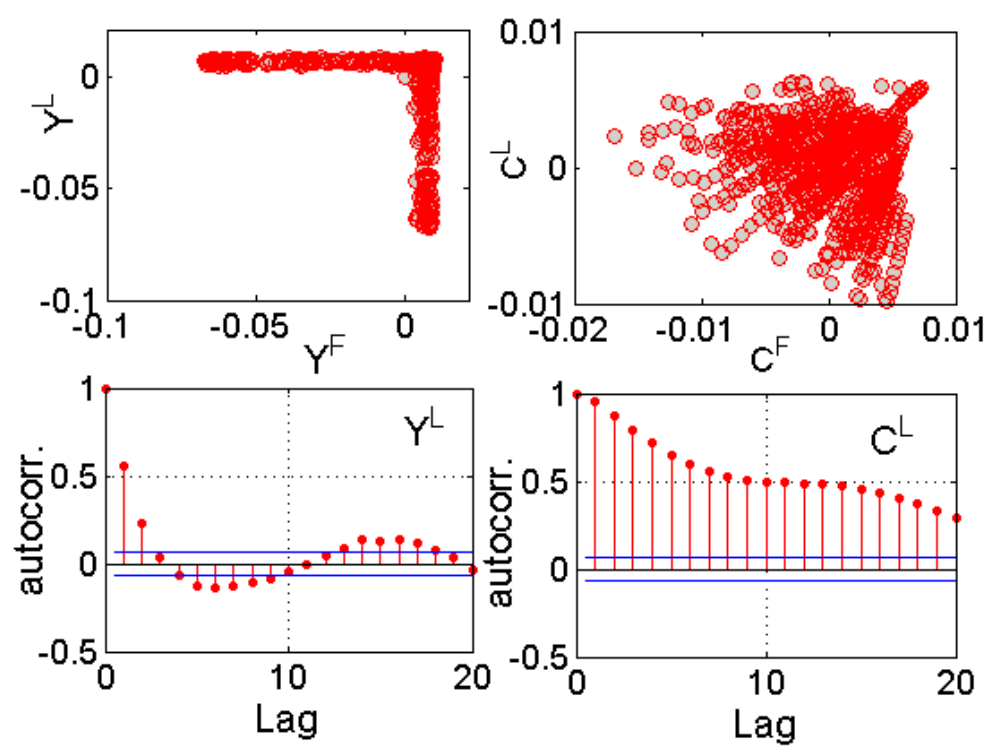

Figure 2: Time series properties of the model. Upper-Left panel: scatter-plot of output deviations of the leader and the follower. Upper-right panel: scatter-plot of consumption deviations of the leader and the follower. Due to consumption smoothing over time, this graph shows more dispersion than the corresponding graph for output. Lower-left panel: autocorrelation structure of output. Lower-right panel: same for consumption. Again, the longer persistence is due to the consumption smoothing motive.

\section{Behavior of the macro variables}

The benefit of using this sort of models is that they allow us to tie the major aggregate variables neatly and study their time series property when subjected to exogenous productivity shocks. However, the drawback is that there is no internal mechanism built into the model which generates interesting time series behavior. In other words, all variables are driven solely by the exogenous shocks and the properties of those shocks are transmitted to these variables. There are other more elaborate models that deals with these kinds of issues (see Ref. [33] for a short review) which we do not pursue here.

Figure 2 plots the response of the model for the tuning parameter $\kappa=-100$. The upper-left quadrant plots the scatter plot of business cycle fluctuations of the leader and the follower country. This property is directly inherited from the structure of the technology evolution (see the lower-right inset of Fig. 1). The upper-right panel shows the scatter plot of consumption response to the technology shocks. Note that the consumption pattern is very different from that of output. This is because of consumption-smoothing property of the agents in the economy. Capital formation provides a channel to smooth the consumption stream and hence, it does not respond moderately to technology shocks. The lower-left panel shows the autocorrelation structure of the GDP of the leading economy. The follower also shows a similar behavior. It is interesting to see the similarity of the autocorrelation behavior of the actual real world countries (Fig. 3). Given that the data set (Ref. [34]) contains

yearly data for 54 years only, some spurious correlation might be present at large values 

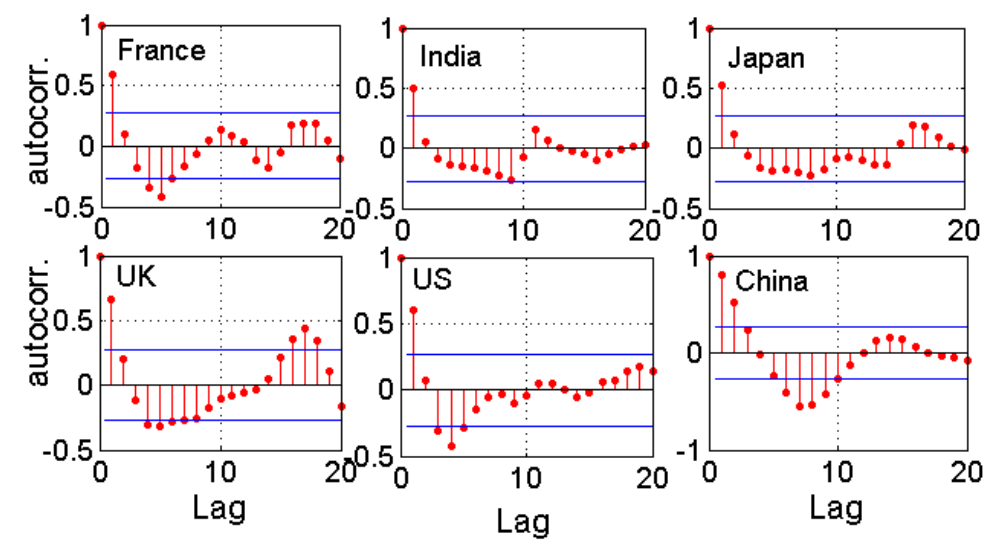

Figure 3: Autocorrelation structure of the business cycle component of per-capita output in a sample of countries. It is noteworthy that all of the countries have non-linear dependence on their past values, developed and emerging countries alike.

of lags. However, this finding is in line with the cross-correlation properties documented in for example, Ref. [35]. The lower-right panel shows the autocorrelation function of the consumption stream. Note that it is far more persistent than the GDP series because the agents respond less to fluctuations and smooth consumption over time.

An important factor would be the effects of the tuning parameter $\kappa$ on the dynamics of the model. In Fig. 4, we show three cases of the dynamics of the models for different values of $\kappa$. The cross-correlation structure remains qualitatively similar for the range of values considered and it also clearly demonstrates the spill-over effects as we see that even with substantial lags, the two time-series (for the leader and the follower) have non-trivial correlations. The autocorrelation structure also has parallels with the actual data (Fig. 3). The pattern of autocorrelations with alternating signs indicate non-linear dependence of the present value of the variable considered on its past.

\subsection{Lag-lead structure of booms and busts}

Ref. [35] is a comprehensive study of the lag-lead structure of the countries in the European Union. The model naturally generates a lag-lead structure in real activities. The aggregate variables like GDP, consumption, investment as well as the prices like real wage rate, rental rate follows the upturn and downturn in sync. Since the LV mechanism induces a lagged correlation structure in the evolution of technology, that property is inherited by all other variables. So a boom (bust) in the leading economy will be followed by a boom (bust) in the follower. Note that even though the economies are in sync with each other at a certain lag, individually they follow stochastic paths as the state of technology hits the economy at random intervals. 

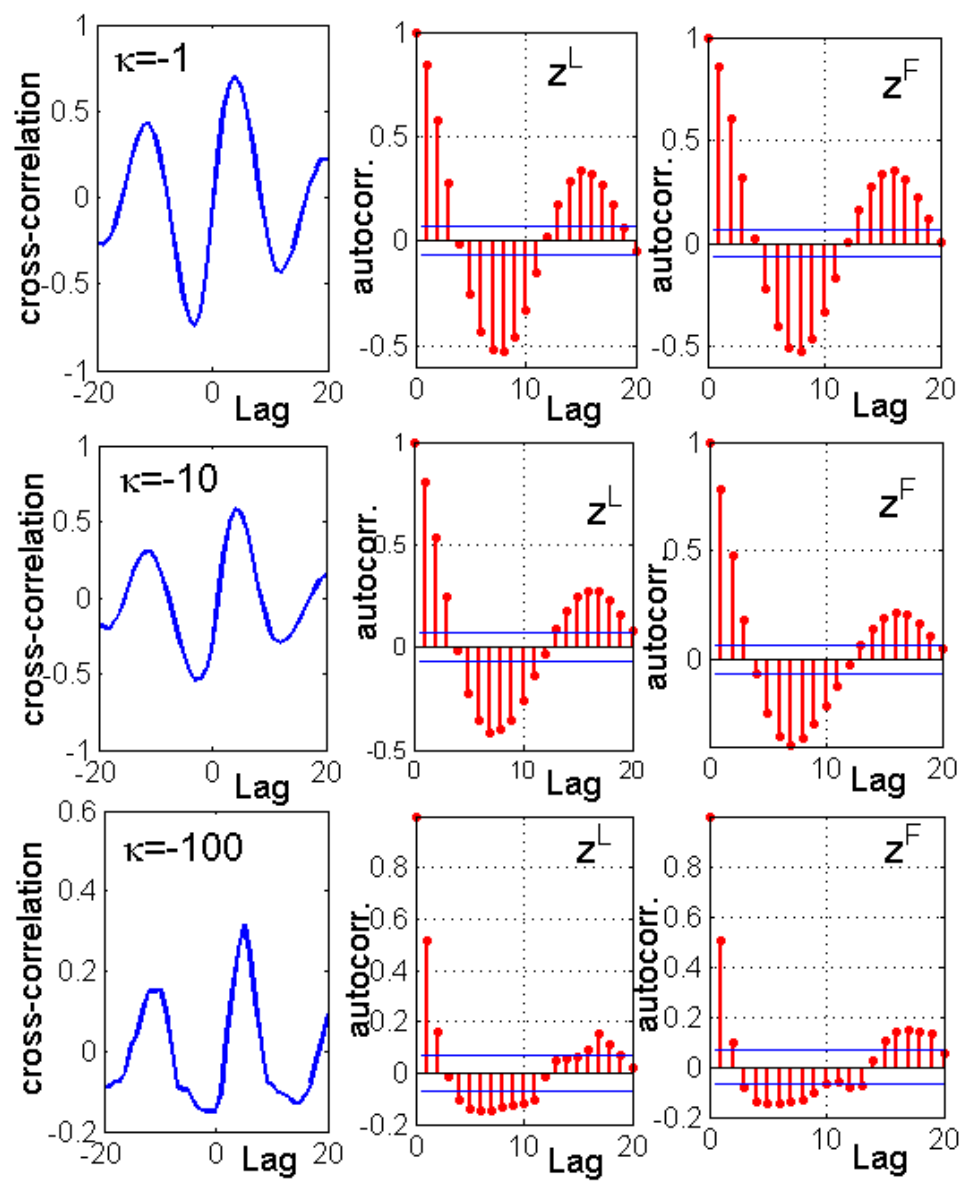

Figure 4: Correlation structure of the technology path for different values of $\kappa$ which controls the standard deviation of the stochastic process. Left column: Cross-correlation between the leader and the follower. Middle column: autocorrelation for the leader. Right column: same for the follower. In three rows, we show three cases, viz. $\kappa=-1,-10,-100$. The basic cross-correlation and the autocorrelation pattern remains qualitatively similar for technology processes with different standard deviation. The cross-correlation patterns clearly shows the lagging effect of spill-overs of booms and busts from the leader to the follower. The autocorrelation pattern shows alternating signs at different lags as in detrended per capita output series. 


\section{Summary}

We have presented a model of technology evolution by applying the LV type nonlinear interaction model that generates endogenous fluctuations. When coupled with a business cycle framework, we show that this mechanism provides a way to capture cross-correlation among countries. In particular, the lagged correlation structure between the leader in the technology frontier and the follower is easily pinned down and shown with help of simulation. As such, the multi-species competition models provide a way to think about countries in the current world competing against each other along with different institutions making them differently able to create or absorb innovations (Ref. [21]).

For the analysis of individual countries, we see that they have an autocorrelation structure close to what is found in real data. Instead of a monotonic decay, it fluctuates a lot before converging to zero. This feature has been found and addressed before in the business cycle literature (Ref. [23], [24]). The present work provides a complementary view on the problem.

A direct mapping between how actual competition takes place in the real economy and the LV mechanism has an issue. It appears in this case that the leader is spending resources to develop a particular technology (or blue-print) for a production process, which is then made available for free to the follower countries. In reality, many companies producing the new technologies make huge profits by selling them to other countries. Thus it may be argued that the potential benefits of being at the forefront outweighs the losses due to investment and the follower countries remain behind all the time. Thus the advanced countries are not 'preys'. While this criticism is correct, it is essentially a definitional issue. The terms 'preys' and 'predators' are used for descriptive purposes. Actually, the fact that less advanced countries remain behind is captured well through the time-series properties as the model clearly shows how spill-over effects from the leader induce fluctuations in the followers. Interestingly Ref. [36] considered a mathematical system to describe competitive interaction between species (as opposed to the predator-prey interaction considered here) and showed that the number of competing species is large enough, then a vast range of dynamical behavior is possible in the system including but not limited to endogenous oscillation and chaos. Although it lies beyond the scope of the present paper, it might provide a plausible alternative.

Another important factor that lends credibility to the application of the LV model is that the fluctuations are endogenous. While there are multiple reasons why economies show fluctuations (e.g. technology shocks, monetary shocks, fiscal shocks, preference shocks etc.), most of the standard models assume exogenous stochastic process to imitate the data. In this case, both the fluctuation and the spill-overs are endogenous. In this regards, we should also mention that there is a stream of literature based on the Goodwin model (Ref. [37], [38]) that shows that endogenous oscillation is possible. The current approach complements that literature in terms of dynamic behavior. A comparison with the Goodwin model also shows that those models are not micro-founded and based on certain equalities and identities linking a number of macro variables. Also this class of models cannot be compared with data easily. The present paper embeds the dynamical system into a clearly micro-founded set up with completely rational agents optimizing over infinite future. Thus we avoid the criticism routinely leveled against Goodwin type models that the assumptions regarding the agents'

behavior are ad-hoc. Still we retain the dynamical features and show that standard tools 
can be applied and the model can be taken to actual data.

The LV system of equations have been used to study multiple features of stock markets (Ref. [17]). Here, we show that it can be used to model technology diffusion in a manner consistent with data. Further explorations would be useful in terms of calibration and potentially to predict downturns of the economies following the leaders due to spill-over effects.

\section{Appendix}

Here we present a detailed discussion on data sources, de-trending techniques and an algorithm to solve the model.

\section{$5.1 \quad$ Data}

To find out the business cycle component of output, we use an HP filter. A standard practice is to decompose GDP per capita into two parts, business cycle and the trend component. The second component is discarded as we do not focus on long-run behavior of the economy. The data is obtained from Ref. [34] database that is freely available in the web. For each country, the database contains data on GDP per capita at yearly frequency, the earliest year being 1960. In principle, one can also use quarterly data without any major quantitative and/or qualitative changes in the results.

Filtering the data The per-capita GDP data shows two features, (1) it usually grows over time indicating a positive trend and (2) it shows fluctuations around the trend. Since

we are concerned about the fluctuations, we first de-trend the real data. Suppose we denote $\log$ of income series by $\left\{y_{t}\right\}$. Then

$$
y_{t}=g_{t}+c_{t}
$$

where $g_{t}$ is the trend component and $c_{t}$ is the cyclical component. Essentially the HP filter solves for an estimation of $g_{t}$ by optimizing a penalized problem. The penalty parameter (chosen by the programer) determines the smoothness of the trend. Once that is determined, the cyclical component is estimated by solving (in vector notation)

$$
\left\{c_{t}^{e s t}\right\}=\left\{y_{t}\right\}-\left\{g_{t}^{e s t}\right\}
$$

There are other filters like BK filter, but the standard practice to de-trend the data is to use the HP filter. So that is what we use. The results presented do not depend crucially on the method chosen for de-trending.

\subsection{Calibration}

The model presented in conjunction with the LV equations is the most basic business cycle model. For calibration purpose, we have assumed the following parameter values: the discount rate $(\beta)=0.99$, capital intensity in the production function $(\theta)=0.36$, depreciation rate $(\delta)=0.025$ and weight assigned to leisure in the utility function $(A)=1.72$. These values are fairly standard for calibration purpose (Ref. [33]). To solve the model numerically, 
one can use software like Dynare (freely available in internet as an add-on to Matlab) or just solve and simulate the forward looking set of equations (Eqn. 8). A more technical point is that while introducing the technology shocks to simulate the time path, we used deviation from the steady state value to feed into the model so that fluctuations in variables are measured in terms of deviations from their respective steady states. Therefore, the shocks would be expressed as

$$
\lambda^{j}(t)=z^{j}(t)-\left\langle z^{j}(t)\right\rangle \text { for } j \in\{L, F\} .
$$

\subsection{Solving the model}

In this section, we describe the algorithm for solving the model. For a comprehensive discussion, see Ref. [33]. Mathematically, we first log-linearize Eqn. 8 around the steady state. Then deviations of all variables from their respective steady state values are linearly related to each other.

\subsection{1 log-linearization}

Essentially for any variable $x_{t}$ in a dynamical equation, we substitute it by

$$
x_{t}=\bar{x} e^{\tilde{x}_{t}}
$$

where $\tilde{x}_{t}$ is the log-deviation,

$$
\tilde{x}_{t}=\log x_{t}-\log \bar{x} .
$$

After substitution, we expand each variable by applying the formula

$$
e^{\theta}=1+\theta \quad \text { where } \theta \rightarrow 0
$$

After cancellation of common terms on both sides, we are left with the reduced form equations describing the log-deviations of all variables in linear forms.

\subsubsection{Solution of the forward-looking system of equations}

For each economy in general, we end up with a system of equations of the following form $(z$ is the state of technology):

$$
\begin{aligned}
A_{1} x_{t}+A_{2} x_{t-1}+A_{3} y_{t}+A_{4} \tilde{z}_{t} & =0 \\
E_{t}\left[B_{1} x_{t+1}+B_{2} x_{t}+B_{3} x_{t-1}+B_{4} y_{t+1}+B_{6} y_{t}+B_{7} \tilde{z}_{t+1}+B_{8} z_{t}\right] & =0 \\
\tilde{z}_{t+1} & =C \tilde{z}_{t}+\epsilon_{t}
\end{aligned}
$$

where $x_{t}=\tilde{K}_{t+1}, y_{t}=\left.\{\tilde{Y}, \tilde{C}, \tilde{H}, \tilde{r}\}\right|_{t}$ and $A_{1, \ldots 4}, B_{1, \ldots 8}, C$ are appropriately defined matrices. The last equation is an $\mathrm{AR}(1)$ approximation of the LV process. This system of equations can be solved in the form

$$
\begin{aligned}
& x_{t}=D_{1} x_{t-1}+D_{2} \tilde{z}_{t}, \\
& y_{t}=D_{3} x_{t-1}+D_{4} \tilde{z}_{t}
\end{aligned}
$$

where $D_{1, \ldots 4}$ are appropriately defined matrices. 


\subsubsection{An algorithm to solve the model}

Here we provide a step by step algorithm.

1. Simulate a baseline LV system for the leader $L$ and follower $F$ (Eqn. 6). Store the time-series of technology generated. Let us call it $\left\{Z^{L}, Z^{F}\right\}$.

2. Sample the time-series with randomly distributed intervals. For the present case, I use uniform distribution. Let us denote the sampled time-series $\left\{S^{L}, S^{F}\right\}$.

3. Transform the sampled time-series by Eqn. 7. This forms the series of technology shocks. The parameter $\kappa$ controls standard deviation of the series.

4. Solve the basic model to get a set of dynamic equations, viz. Eqn. 8.

5. Log-linearize it around the steady state (see App. 5.3.1 above) and generate Eqn. 16 as in App. 5.3.2.

6. Feed the series of technology shocks constructed above into Eqn. 16. This will generate time-series of output $(Y)$, consumption $(C)$ etc. for both the leader and the follower.

After we get the output series $\left\{Y^{L}, Y^{F}\right\}$, we can study the cross-correlation as well as autocorrelation properties at various lags.

\section{References}

[1] R. Mantegna and E. Stanley, Introduction to Econophysics: Correlations and Complexity in Finance. Cambridge University Press, 2007.

[2] S. Sinha, A. Chatterjee, A. Chakraborti, and B. K. Chakrabart, Econophysics: An Introduction. Wiley-VCH, 2010.

[3] X. Gabaix, "Granular origin of aggregate fluctuations," Econometrica, vol. 79-3, 2011.

[4] R. Albert and A.-L. Barabasi, "Statistical mechanics of complex networks," Review of modern physics, vol. 74, pp. 47-97, 2002.

[5] A. Barrat, M. Barthelemy, and A. Vespignani, Dynamical Processes on Complex Networks. Cambridge University Press, 2008.

[6] M. Jackson, Social and Economic Networks. Princeton University Press, 2010.

[7] F. Vega-Redondo, Complex Social Networks. Cambridge University Press, 2007.

[8] S. N. Dorogovtsev and J. F. F. Mendes, Evolution of Networks: From Bilogical Nets to the Internet and $W W W$. Oxford University Press, 2003.

[9] F. Schweitzer, G. Fagiolo, D. Sornette, F. Vega-Redondo, A. Vespignani, and D. R. White, "Economic networks: The new challenges," Science, vol. 325, no. 5939, pp. 422$425,2009$. 
[10] S. Sinha, "Are large complex economic systems unstable?," Science and culture, vol. 76, pp. 454-458, 2010.

[11] E. Atalay, A. Hortascu, J. Roberts, and C. Syverson, "Network structure of production," PNAS, vol. 108, no. 13, p. 51995202, 2011.

[12] R. May, Stability and Complexity in Model Ecosystems. Princeton University Press, 1973.

[13] M. Nowak, Evolutionary Dynamics: Exploring the Equations of Life. Belknap Press, 2006.

[14] J. Hofbauer and K. Sigmund, Evolutionary games and population dynamics. Cambridge University Press, 1998.

[15] S. Solomon, "Stochastic lotka-volterra systems of competing auto-catalytic agents lead generically to truncated pareto power wealth distribution, truncated levy distribution of market returns, clustered volatility, booms and crashes," in Decision Technologies for Computational Finance (A. P. Refenes, A. N. Burgess, and J. E. Moody, eds.), Kluwer Academic Publishers, 1998.

[16] S. Solomon, "Generalized lotka volterra (glv) models of stock markets," in Applications of Simulation to Social Sciences (G. Ballot and G. Weisbuch, eds.), Hermes Science Publications, 2000.

[17] O. Malcai, O. Biham, P. Richmond, and S. Solomon, "Theoretical analysis and simulations of the generalized lotka-volterra model," Physical Review E, vol. 66, p. 031102, 2002.

[18] S. Sinha and S. Sinha, "Evidence of universality for the may-wigner stability theorem for random networks with local dynamics," Physical Review E, vol. 020902, 2005.

[19] S. Sinha and S. Sinha, "Robust emergent activity in dynamical networks," Physical Review E, vol. 066117, 2006.

[20] Lotka-Volterra Equations, "http://mathworld.wolfram.com/lotka-volterraequations. html.".

[21] D. Acemoglu and J. A. Robinson, "Asymmetric growth and institutions in an interdependent world." Working paper, 2014.

[22] T. Di Matteo, T. Aste, and M. Gallegati, "Innovation flow through social networks: productivity distribution in france and italy," The European Physical Journal B, vol. 47 (3), pp. 459-466, 2005.

[23] S. Schmitt-Grohe, "Endogenous business cycles and the dynamics of output, hours, and consumption," American economic review, vol. 90 (5), pp. 1136-1159, 2000.

[24] T. Cogley and J. Nason, "Output dynamics in real-business cycle models," The American Economic Review, vol. 85 (3), pp. 492-511, 1995. 
[25] B. Podobnik, D. H. D. Y. Kenett, and H. E. Stanley, "The competitiveness versus the wealth of a country," Nature Scientific Reports, vol. 2, p. 678, 2012.

[26] J. Tenenbaum, D. Horvatic, S. C. Bajic, B. Pehlivanovic, B. Podobnik, and H. E. Stanley, "Comparison between response dynamics in transition economies and developed countries," Physical Review E, vol. 82, p. 046104, 2010.

[27] M. H. R. Stanley, L. A. N. Amaral, S. V. Buldyrev, S. Havlin, H. Leschhorn, P. Maass, M. A. Salinger, and H. E. Stanley, "Scaling behavior in the growth of companies," Nature, vol. 379, pp. 804-806, 1996.

[28] D. Canning, L. A. N. Amaral, Y. Lee, M. Meyer, and H. E. Stanley, "A power law for scaling the volatility of gdp growth rates with country size," Economics Letters, vol. 60, pp. 1385-1388, 1998.

[29] J. Shao, P. C. Ivanov, B. Urosevic, H. E. Stanley, and B. Podobnik, "Zipf rank approach and cross-country convergence of incomes," Europhysics Letters, vol. 94, p. 48001, 2011.

[30] M. Gallegati and M. Gallegati, "Wavelet variance analysis of output in G-7 countries," Studies in Nonlinear Dynamics $\&$ Econometrics, vol. 11 (3), pp. 1-25, 2007.

[31] M. Gallegati, M. Gallegati, and W. Polasek, "Business cycle fluctuations in mediterranean countries (1960-2000)," Emerging Markets Finance and Trade, vol. 40 (6), pp. 28-47, 2004.

[32] D. Acemoglu, Introduction to Modern Economic Growth. Princeton University Press, 2009.

[33] G. McCandless, "The abcs of rbcs," Harvard University Press, 2008.

[34] World Bank, "http://data.worldbank.org/."

[35] A. M. Agresti and B. Mojon, "Some stylized facts on the euro area business cycle," in Monetary Policy Transmission in the Euro Area, Eds: I. Angeloni and A. Kashyap and B. Mojon (CUP), 2003.

[36] S. Smale, "On the differential equations of species in competition," Journal of Mathematical Biology, vol. 3, pp. 5-7, 1976.

[37] R. M. Goodwin, "A growth cycle," in Socialism, capitalism and economic growth (C. H. Feinstein, ed.), Cambridge University Press, 1967.

[38] R. M. Goodwin, Chaotic economic dynamics. Oxford University Press, 1990. 